\title{
Large-Scale, High-Resolution Neurophysiological Maps Underlying fMRI of Macaque Temporal Lobe
}

\author{
Elias B. Issa, ${ }^{1}$ Alex M. Papanastassiou, ${ }^{1,2}$ and James J. DiCarlo ${ }^{1}$ \\ ${ }^{1} \mathrm{McGovern}$ Institute for Brain Research and Department of Brain and Cognitive Sciences, Massachusetts Institute of Technology, Cambridge, \\ Massachusetts 02139, and 'Brigham and Women's Hospital and Children's Hospital, Harvard Medical School, Boston, Massachusetts 02115
}

\begin{abstract}
Maps obtained by functional magnetic resonance imaging (fMRI) are thought to reflect the underlying spatial layout of neural activity. However, previous studies have not been able to directly compare fMRI maps to high-resolution neurophysiological maps, particularly in higher level visual areas. Here, we used a novel stereo microfocal x-ray system to localize thousands of neural recordings across monkey inferior temporal cortex (IT), construct large-scale maps of neuronal object selectivity at subvoxel resolution, and compare those neurophysiology maps with fMRI maps from the same subjects. While neurophysiology maps contained reliable structure at the submillimeter scale, fMRI maps of object selectivity contained information at larger scales $(>2.5 \mathrm{~mm})$ and were only partly correlated with raw neurophysiology maps collected in the same subjects. However, spatial smoothing of neurophysiology maps more than doubled that correlation, while a variety of alternative transforms led to no significant improvement. Furthermore, raw spiking signals, once spatially smoothed, were as predictive of fMRI maps as local field potential signals. Thus, fMRI of the inferior temporal lobe reflects a spatially low-passed version of neurophysiology signals. These findings strongly validate the widespread use of fMRI for detecting large ( $>2.5$ $\mathrm{mm}$ ) neuronal domains of object selectivity but show that a complete understanding of even the most pure domains (e.g., faces vs nonface objects) requires investigation at fine scales that can currently only be obtained with invasive neurophysiological methods.
\end{abstract}

\section{Introduction}

As a noninvasive measure of whole brain activity, fMRI has become invaluable in studying human brain function. However, fMRI is only an indirect measure of neural activity-it depends on the potentially complex coupling between the neural events of interest and their temporally lagged, indirect influence on vascular events (Attwell et al., 2010; Kriegeskorte et al., 2010; Masamoto and Kanno, 2012). A previous study found that neural and fMRI signals were correlated over time at a given location (Logothetis et al., 2001) but did not measure whether the pattern of selectivity in fMRI across space corresponded to the underlying pattern in neurophysiologyan important remaining question given the widespread use of fMRI for inferring the 3D layout of brain function.

Studies in primary visual cortex (V1) have suggested that the spatial spread of fMRI signals is $2-4 \mathrm{~mm}$ [full-width at half-

Received March 22, 2013; revised July 19, 2013; accepted Aug. 13, 2013.

Author contributions: E.B.I., A.P., and J.J.D. designed research; E.B.I. and A.M.P. performed research; E.B.I. analyzed data; E.B.I. and J.J.D. wrote the paper.

This research was supported by US National Eye Institute grants R01-EY014970 (J.J.D.) and K99-EY022671 (E.B.I.), The McGovern Institute for Brain Research, and an NIH NRSA postdoctoral fellowship F32-EY019609 (E.B.I.). We thank J. Deutsch, K. Schmidt, and P. Aparicio for help with MRI and animal care, B. Andken and D. Oreper for help with $\mathrm{x}$-ray software and hardware, and B. Andken and C. Stawarz for help with experiment software. We thank N. Kanwisher for helpful comments and discussion on this manuscript.

The authors declare no conflict of interest.

Correspondence should be addressed to Elias Issa, McGovern Institute for Brain Research, Department of Brain and Cognitive Sciences, 77 Massachusetts Institute of Technology, 46-6157, Cambridge, MA 02139. E-mail: issa@mit.edu.

A. Papanastassiou's present address: Department of Neurosurgery, University of Texas Health Science Center, San Antonio, TX 78229.

DOI:10.1523/JNEUROSCI.1248-13.2013

Copyright $\odot 2013$ the authors $\quad 0270-6474 / 13 / 3315207-13 \$ 15.00 / 0$ maximum (FWHM)] based on inference from previously known functional organization in V1 (Engel et al., 1997; Parkes et al., 2005; Olman et al., 2007). However, these studies did not compare neurophysiology to fMRI directly in the same subjects (Disbrow et al., 2000; Kim et al., 2000; Duong et al., 2001; Kim et al., 2004; Fukuda et al., 2006; He et al., 2008; Yacoub et al., 2008; Bell et al., 2011), which may be required in studying higher level areas such as inferior temporal cortex (IT) where neural spatial organization is not as well understood as in V1 (Hubel and Wiesel, 1974; Ohki et al., 2005) and where there may be more variability over individuals. A main methodological limitation has been that previous neurophysiology studies relied on coarse, extrapolative electrode localization techniques (Tsao et al., 2006; Bell et al., 2011) that do not support quantitative, fine-scale construction of neural maps for comparison to fMRI. Although optical imaging techniques have provided exquisite neural maps, they are limited to surface-accessible cortex and to imaging windows of a few millimeters (Wang et al., 1996; Tanigawa et al., 2010), whereas parts of IT cortex are buried in a major cortical sulcus or at the base of the skull and are thus invisible to these methods.

Here, we aimed to map the spatial organization present at the neural level in macaque IT, an area thought to contain object selective domains, to establish a neurophysiological gold standard for comparison to previously measured fMRI maps (Op de Beeck et al., 2008; Bell et al., 2009; Pinsk et al., 2009; Tsao et al., 2003, 2008a; Ku et al., 2011). Utilizing a novel stereo microfocal $\mathrm{x}$-ray system for electrode localization (Cox et al., 2008), we obtained subvoxel ( $\sim 300 \mu \mathrm{m}$ resolution), in vivo estimates of electrode position while widely sampling recording sites across the whole of inferior temporal cortex $(\sim 15 \times 10 \times 3 \mathrm{~mm})$ surpassing 
Table 1. Comparison of conditions in fMRI and neurophysiology experiments

\begin{tabular}{lll}
\hline & fMRI & Neurophysiology \\
\hline Age (years) & $4-6$ & $8-10$ \\
Weight (kg) & $3-4$ & $5-7$ \\
Presentation mode & Block & Interleaved \\
Image duration (ms) & 300 & 100 \\
Presentation rate (images/s) & 1.4 & 5 \\
Image size (deg) & 6 & 6 \\
\# of sampled locations (M1) & 159 voxels & 389 sites \\
\# of sample locations (M2) & 340 voxels & 1522 sites \\
\hline
\end{tabular}

fMRI experiments were conducted separately from neurophysiology experiments following methodologies used in prior work (Tsao et al., 2003; Hung et al., 2005). This introduced some differences in the collection of fMRI and neurophysiology data. For example, images were presented at slower rates $(1.4 \mathrm{~Hz})$ in $\mathrm{fMRl}$ experiments as opposed to neurophysiology experiments $(5 \mathrm{~Hz})$, and fMRI data were collected when monkeys were younger in age. A detailed explanation of similarities and differences in fMRI and neurophysiology is provided in Materials and Methods.

the coverage-resolution tradeoffs inherent in previous work (Wang et al., 1996; Bell et al., 2011). We found that neurophysiology maps contained reproducible structure at submillimeter spatial scales but were only weakly predictive of fMRI maps. However, spatially smoothed versions of the neurophysiology maps correlated quite well with fMRI maps. This finding provides empirical validation of the utility of current fMRI methodology in revealing large scale object selective structures in IT while also demonstrating its current limitations for discriminating the finer neural structure that is present in IT cortex.

\section{Materials and Methods}

\section{Animals and surgery}

Two rhesus macaque monkeys (Macaca mulatta) weighing 3-4 kg at the time of scanning and 5-7 kg at the time of electrophysiological recording $(\mathrm{M} 1=$ male, $\mathrm{M} 2$ = female; referred to as monkey J and monkey $\mathrm{M}$, respectively, in previous fMRI work) (Op de Beeck et al., 2008) were used in this study. Monkeys were scanned at five years of age after reaching sexual maturity and after adult brain size is typically reached (Malkova et al., 2006), and physiological recordings were performed three years later. Despite the large gap in time between imaging and physiology experiments, anatomical scans revealed almost no longitudinal change in brain size during the intervening period and correlation of structural MR volumes across this time period was $\sim 0.8$ in the temporal lobe of both monkeys.

Details of headpost and recording chamber implant surgeries are described elsewhere (Issa and DiCarlo, 2012). Briefly, before behavioral training and fMRI scanning, a plastic, fMRI-compatible headpost was implanted in dental acrylic anchored to the skull using ceramic screws. In a second surgery before electrophysiological recording, a plastic, cylindrical chamber was implanted for targeting recordings to the temporal lobe using a dorsal to ventral approach. All procedures were performed in compliance with National Institutes of Health guidelines and the standards of the MIT Committee on Animal Care and the American Physiological Society.

\section{Images and behavioral training}

Because fMRI and neurophysiology experiments were conducted separately, image presentation and task conditions varied slightly. For both fMRI and neurophysiology, we followed standard methodologies used in previous studies (Tsao et al., 2003; Hung et al., 2005). Here, we provide an integrated summary of the similarities and differences between experiments (also see Table 1). In both experiments, presented visual images contained a single object drawn from six possible categories, monkey faces, nonface objects, power spectrum matched scrambles of nonface objects, "smoothies," "spikies," and "cubies." The latter three classes were synthetic objects generated in Matlab and are described in our previous work (Op de Beeck et al., 2008). In both experiments, images were presented at a size of six degrees of visual angle. However, an LCD projector was used during scanning $(1920 \times 1200,75 \mathrm{~Hz}$ refresh rate, $\sim 50 \mathrm{~cm}$ in front of the animal) while a CRT monitor was used during neurophysiology $(1900 \times 1200,85 \mathrm{~Hz}$ refresh rate, $55 \mathrm{~cm}$ in front of animal). In fMRI experiments, monkeys were trained to fixate on a central fixation square ( 0.3 degree diameter) within a \pm 1.25 degree fixation window while a \pm 2 degree fixation window was used in physiology experiments; however, in post hoc analyses we found that monkeys generally maintained fixation within a much smaller 1 degree region and do not scan across images [Issa and DiCarlo (2012), their Fig. 5A]. During fMRI sessions, images were presented in block design such that image exemplars drawn from a single category were presented in a single block at slow presentation rates $(300 \mathrm{~ms}$ on, $420 \mathrm{~ms}$ off or $1.4 \mathrm{~Hz}$ ). In physiology experiments, images from all categories were randomly interleaved (10 or 20 exemplars each for faces, objects, and scrambled objects and 3 or 20 exemplars each for smoothies, spikies, and cubies) at much faster rates (rapid serial visual presentation: $100 \mathrm{~ms}$ on, $100 \mathrm{~ms}$ off or $5 \mathrm{~Hz}$ ) (Hung et al., 2005; De Baene et al., 2007). In physiology experiments, each fixation trial lasted three to five seconds (15-25 images) while in fMRI experiments fixations lasted tens of seconds during category blocks lasting $45 \mathrm{~s} \mathrm{(} \sim 60$ images). Although stimulus presentation conditions were not matched between electrophysiology (interleaved, rapid presentation) and fMRI (blocked, slower presentation), previous work has shown that rank order neural object selectivity is similar across both fast and slow stimulus presentation rates (Edwards et al., 2003; De Baene et al., 2007). Furthermore, similar category selective regions are observed under fMRI using both blocked and interleaved (event-related) designs (Kanwisher et al., 1997; Mur et al., 2012; Vul et al., 2012).

Each scan run contained nine blocks lasting a total of $7 \mathrm{~min}$. Runs included three blocks each of three categories (faces, objects, scrambled objects or smoothies, spikies, cubies). Since these two run types (natural and synthetic) were collected on different days, maps were constructed for contrasts within runs (e.g., smoothies minus spikies) and not across runs (e.g., faces minus smoothies), yielding six total contrasts per subject. fMRI data were collected for multiple runs across three to seven scan sessions ( $\sim 30$ total runs in $\mathrm{M} 1 ; \sim 50$ total runs in $\mathrm{M} 2)$.

In monkey M2, many sites were not tested with all six categories leading to variable number of sites per contrast in M2 (faces and objects, $n=$ 1522; scrambled objects, $n=229$; smoothies/spikies/cubies, $n=450$ ) while all sites in monkey M1 were tested in this study with all six categories $(n=369)$. Also, in monkey M2, fewer exemplars were used per category, but at least 15 presentations ( 3 images $\times 5$ repetitions) were collected per category and many more presentations were obtained in most cases across both monkeys (median $=57$ trials/category).

\section{Functional imaging}

Functional scanning was conducted at the Martinos Center for Biomedical Imaging at Massachusetts General Hospital in a horizontal bore 3 tesla Siemens Tim Trio scanner using a surface coil. A contrast agent [monocrystalline iron oxide nanoparticle (MION)] was injected intravenously before scanning. Functional scans were acquired using a gradient echo planar (EPI) sequence with coronal slice prescription $(1.375 \mathrm{~mm}$ slice thickness). In addition to functional scans, T1-weighted anatomical scans were obtained before functional imaging experiments and before neurophysiology experiments. Additional details of functional and anatomical scans can be found in our previous studies (Op de Beeck et al., 2008; Issa and DiCarlo, 2012).

\section{Neurophysiological recordings}

In both monkeys, neurophysiological recordings were obtained in the left hemisphere. Multiunit activity (MUA) and local field potentials (LFPs) were recorded as described previously using glass-coated tungsten microelectrodes (M1 $=389, \mathrm{M} 2=1522$ total sites) (Issa and DiCarlo, 2012). Penetrations traveled vertically from dorsal to ventral, and neurophysiological data were collected systematically at 300 to $500 \mu \mathrm{m}$ intervals sampling mostly gray matter locations but also some white matter locations (see example coronal slices in Fig. 1A). More extensive sampling of posterior IT was obtained in monkey M2 (see Figs. 3, 4) while in monkey M1, sampling was restricted to the anterior two-thirds of IT (see Fig. 5). The raw electrode signal ( $1 \mathrm{~Hz}$ to $4 \mathrm{kHz}, 8 \mathrm{kHz}$ sampling rate) was stored, and the LFP was obtained by lowpass filtering $(<300 \mathrm{~Hz}, 128$ order elliptical filter) the raw electrode signal and notch filtering at $60 \mathrm{~Hz}$ and its harmonics $(120,180$, and $240 \mathrm{~Hz})$. 


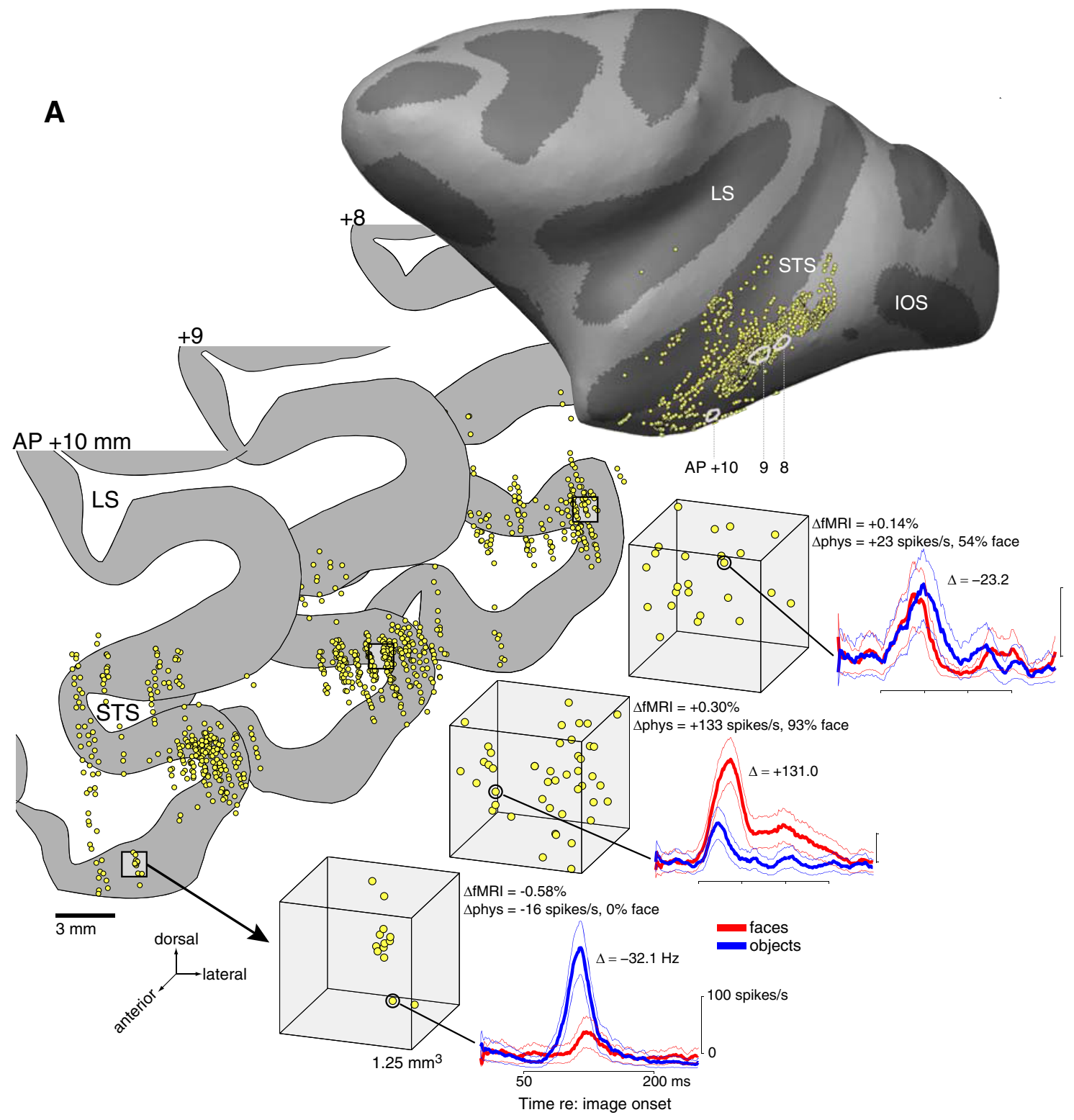

B

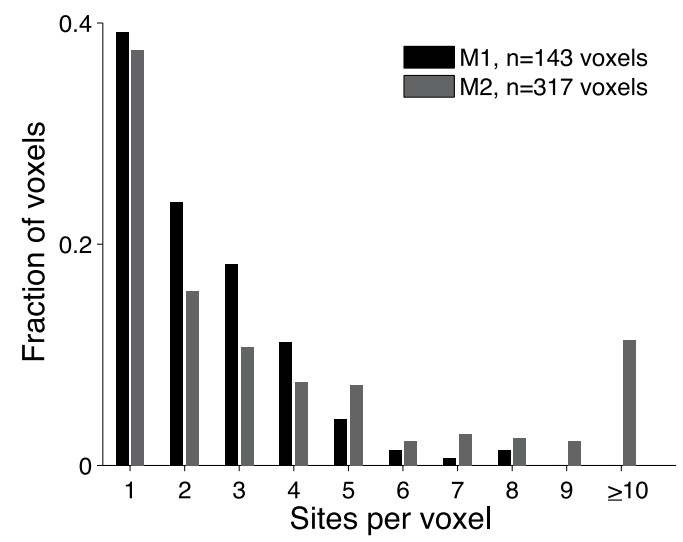

Figure 1. Neurophysiological mapping of IT cortex. $A$, Locations of neurophysiological recordings in subject $M 2$ are shown on an inflated surface view of the brain. The locations of three example voxels are projected to their approximate locations on the surface (gray outlines). Locations spanned the posterior to anterior extent of IT ( $20 \mathrm{~mm}$ ) (cortical gray matter-white matter boundary shown; dark regions indicate sulci and light regions gyri; sites are projected from 3D to the nearest point on the 2D gray-white manifold; STS, superior temporal sulcus; LS, lateral sulcus; I0S, inferior occipital sulcus). The coronal sections demonstrate the locations of the physiology sites relative to the cortical gray matter (gray ribbon) as derived from T1-weighted anatomical scans and relative to example fMRI voxels (light shaded squares). Furthermore, the relative 3D positions of individual sites within three example voxels are shown. For each site, the response to faces and nonface objects was measured, and selectivity was computed as the difference in these responses in a 50-200 ms window post image onset (example poststimulus time (Figure legend continues.) 


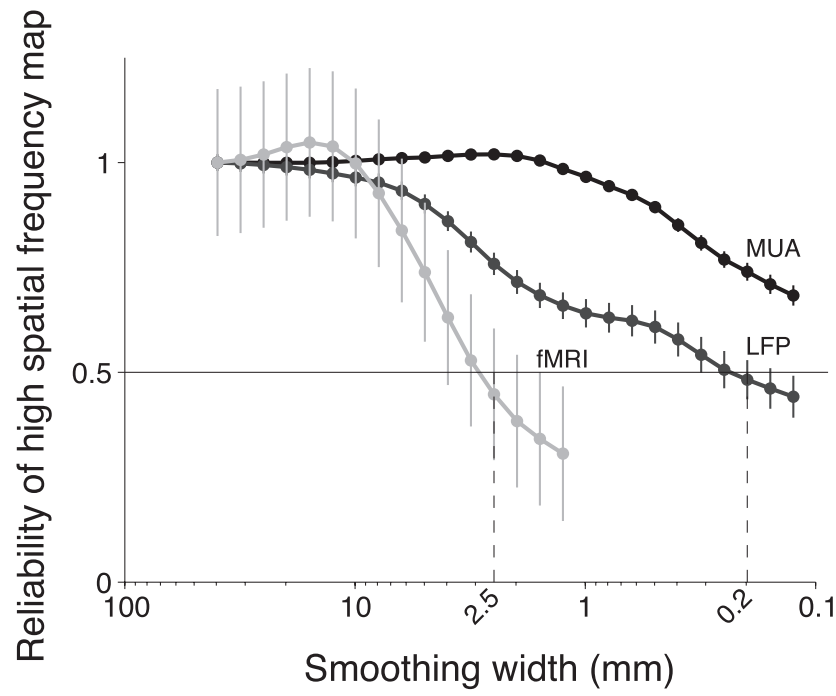

Figure 2. Scale of correlations in neurophysiology and fMRI maps. MUA and LFP maps contained reproducible structure at finer spatial scales $(<1 \mathrm{~mm})$ than fMRI maps $(2.5 \mathrm{~mm})$ (based on smoothing width at $50 \%$ height; vertical lines). To estimate the scale of spatial correlations within a map, maps smoothed at a particular resolution using a Gaussian kernel were subtracted from raw maps. The correlation of the residuals in independent data sets $(n=30$ iterations of split halves data) was used as an estimate of any reliable structure after removing spatial frequency content up to that smoothing width. Smaller smoothing widths (i.e., $0.1 \mathrm{~mm}$, right) remove most spatial information leading to high-passed maps; at the other extreme, large smoothing widths (i.e., $40 \mathrm{~mm}$, left) only remove very low spatial frequencies while retaining most spatial frequencies and thus most of the reliable structure. Note that MUA and LFP correlations reached higher absolute levels than $\mathrm{MMRI}$ maps because fMRI maps were noisier (less reliable across trials) than MUA and LFP maps. However, normalized values are plotted on the ordinate for ease of comparison. fMRI maps did contain some information below $2 \mathrm{~mm}$ spatial scales, but correlations were weak in this regime (error bars reflect SEM across the 12 contrast maps collected). Correlations in fMRI maps were computed along a contiguous set of voxels (volume) encompassing the locations of MUA and LFP sites. MUA and LFP curves are truncated at $100 \mu \mathrm{m}$ because of the limits of the resolution of $x$-ray-based mapping, and the fMRI curve is truncated at $1.25 \mathrm{~mm}$ since this was the voxel resolution used in scanning.

\section{$X$-ray-based electrode localization}

All electrophysiological recordings were visualized using a stereo microfocal $\mathrm{x}$-ray system developed previously in our laboratory (Cox et al., 2008), and details of reconstruction of recording locations in 3D space and coregistration to MRI are described elsewhere (Issa and DiCarlo, 2012). Although the ex vivo resolution of the $\mathrm{x}$-ray system is $\sim 30 \mu \mathrm{m}$, the in vivo accuracy is limited by factors such as tissue movement either from electrode forces or from brain movement across days. Based on preliminary testing using internal fiducials to monitor movement, these sources of motion error are on the order of a few hundred micrometers (Issa et al., 2010) and are not a limiting factor in comparisons to fMRI maps that were $>2.5 \mathrm{~mm}$ in effective resolution (Fig. 2).

Analysis

MUA, LFP, and fMRI responses. For fMRI data, linear response weights were estimated using a general linear model (GLM) to fit the time series

\section{$\leftarrow$}

(Figure legend continued.) histograms shown on far right). Metrics such as the purity (fraction of sites preferring faces) or the average response differential within a voxel could be computed and compared with the fMRI percentage signal change for the faces versus nonface objects contrast (see text next to each example voxel). In general, quantitative analyses were performed in voxel (volume) space, and surface-based representations (Figs. 3-5) are for qualitative appreciation of the data. $\boldsymbol{B}$, Distribution of the number of sites collected per voxel in the faces versus objects contrast maps measured in both monkeys. In voxels that were sampled for face versus object selectivity, sampling density averaged 4.4 sites per voxel in monkey M2 (36 voxels with $\geq 10$ sites sampled; also, see Fig. $1 \boldsymbol{A}$ ) and averaged 2.3 sites per voxel in monkey M1. For the remaining contrast maps, sampling density averaged $\sim 2$ sites/voxel. in each voxel [see the study by Op de Beeck et al. (2008) for detailed description of fMRI preprocessing and analysis]. Scans from multiple sessions collected before and after $\sim 3$ months of discrimination training for spikies (monkey M1) and smoothies (monkey M2) were motion corrected and registered, and the $\beta$ weights in each voxel were averaged to yield a single volume for each contrast. For the majority of the analyses of this paper, fMRI maps were smoothed using a Gaussian kernel (FWHM $=2.5 \mathrm{~mm}$ ). In our previous work, we showed that smoothing fMRI maps at $2.5 \mathrm{~mm}$ scales was optimal for reducing noise while preserving as much large scale structure as possible [Op de Beeck et al. (2008), see their supplemental Fig. 5]. In the present work, we confirmed that reliable information in fMRI maps drops off sharply at $2.5 \mathrm{~mm}$ suggesting that little information was lost by smoothing fMRI maps (Fig. 2). Furthermore, when we repeated analyses using unsmoothed fMRI data, the main findings did not change (see Results). Multiunit firing rates for each object class were computed by averaging spike counts across all exemplars in a 50-200 ms window following image onset (Fig. 1A). LFP waveforms were first DC corrected, and the root mean square (RMS) amplitude of the average waveform across all image presentations of a category was computed. Category selectivity was measured as the difference in raw spike rates (MUA), RMS power (LFP), or $\beta$ weights (fMRI). Computing a d' or $t$ statistic that normalizes by SD of the signal yielded similar results as using a simple difference of the means that has the added advantage of not relying on the number of trials collected and that as a linear operation allows for commutativity of applied transforms. Sites were included in analysis if their response to either category in a contrast was at least $1.65^{\star}$ SEM different from baseline (baseline computed in the $0-50 \mathrm{~ms}$ window following image onset; for LFPs, the mean of SD in each time bin was used to compute the SEM). In separate analyses that we conducted, distinguishing sites/voxels based on whether they were in white matter or gray matter (see Fig. $6 B$ ) or simply including all sites did not affect the main findings. No additional visual drive criteria were used in selecting fMRI voxels, so fMRI voxels were used in analysis purely based on whether they contained at least one neurophysiology site.

Spearman's rank correlations were computed pointwise between MUA (or LFP) sites and corresponding fMRI voxels. It is important to note that all correlations were computed in native $3 \mathrm{D}$ space such that quantitative comparisons were done in the original fMRI volume (in one control analysis, correlations were done after projecting to the surface; see Fig. $6 B$ ). However, for the purpose of visualization, data are plotted after projecting onto a 2D surface (Figs. 3-5; see Fig. $1 A$ for illustration of this projection). These visualizations are only meant for qualitative evaluation. Surface flattening is not a distance preserving transform (nonisometric) and may exaggerate some distances (e.g., in unfolding a sulcus) while compressing others (e.g., on the gyrus). Furthermore, sites are collapsed to the surface in going from $3 \mathrm{D}$ to $2 \mathrm{D}$, so depth information is lost. These factors alter the spatial correspondence between fMRI and neurophysiology. In the present study, we took the conservative approach of performing quantitative fMRI and neurophysiology comparisons in 3D space.

Neurophysiology-to-fMRI transforms. We applied transforms to neural and/or fMRI maps to test specific hypotheses about the linkage between neurophysiology-determined and fMRI-determined maps. Each transform was tested individually or cascaded with a fixed spatial smoothing transform. Below, we list the 16 types of transforms that we applied (Fig. 6B): (1) Spatial smoothing: A three-dimensional Gaussian kernel was used to smooth neurophysiology maps to different degrees (FWHM $=0$ to $24 \mathrm{~mm}$ ) in native 3D space. (2) Balanced spatial smoothing: Since physiology sites were not uniformly sampled in space, smoothing estimates could be biased by regions where dense sampling was obtained by chance. For example, if only a few sites were collected near to the voxel to be predicted, then predictions based on smoothing might be inadvertently dominated by the large number of distant sites. To ensure that the effects of uneven sampling were not a concern, we performed a control (called "balanced sampling"; Fig. 6B) where we averaged sites in $1 \mathrm{~mm}$ bins before smoothing. This procedure insured that the predicted fMRI response at each voxel was, for example, not dominated by dense samples of physiology sites at distant locations. This correction was performed in 


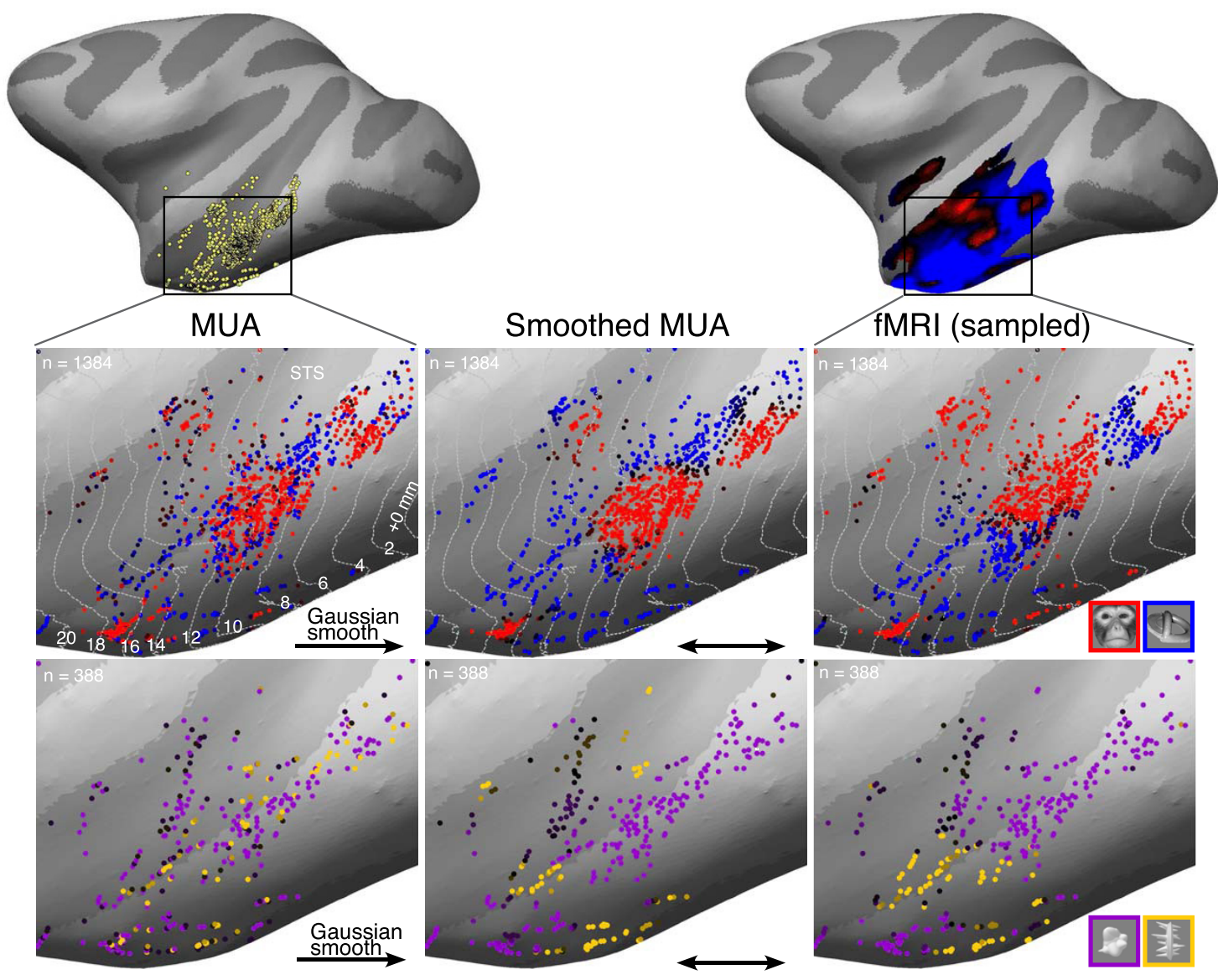

Figure 3. Example neurophysiological maps of category selectivity in IT. Neurophysiological maps of object selectivity (left) appeared more heterogeneous compared with fMRI maps (right) that showed more gradual, large-scale gradients of category selectivity (e.g., note the presence of a large face-selective region, the middle face patch, in central IT). Optimal spatial smoothing yielded neurophysiological maps (middle) that more closely resembled the large-scale structure present in fMRI maps. This improvement was apparent in the smoothie versus spikie map where smoothie and spikie selective regions became more clearly segregated after spatial smoothing (middle) even though smoothie and spikie selective sites were intermixed in raw neurophysiology maps (left). Neurophysiology and fMRI maps were quantitatively compared only at locations where sites were recorded, and fMRI data shown here are plotted as dots at these corresponding locations. It is also important to note that all correlations were computed in native 3D space; surface-based visualization is used here for qualitative visual comparison of contrast maps as surface flattening and projection of sites onto the surface is not a distance preserving transform (non-isometric) and alters the spatial correspondence between fMRI and neurophysiology.

addition to smoothing with a Gaussian spatial kernel, and it is important to note that the form of the Gaussian kernel also acts to enforce the intuition that nearby sites should be weighted more strongly than distant sites. (3) Response windows: Responses in adjacent $50 \mathrm{~ms}$ response windows were tested. (4) d': Mean response differences were normalized by the RMS of the SDs for the two categories tested $\left(\mu_{1}-\mu_{2}\right) / \mathrm{rms}\left(\sigma_{1}, \sigma_{2}\right)$. (5) Contrast: Mean response differences were normalized by the sum of the absolute value of the mean responses $\left(\mu_{1}-\mu_{2}\right) /\left(\left|\mu_{1}\right|+\left|\mu_{2}\right|\right)$. (6) Binarized: Responses were set to -1 or 1 based on category preference. (7) Rectified: If the strongest response in a category pair was inhibitory, then responses were rectified (i.e., if category 1 was more strongly inhibited than category 2 was excited, the negative response differential, $\mu_{1}-$ $\mu_{2}$, was rectified). The rationale behind this transform is that if the fMRI signal is related to synaptic processing, then activity at both inhibitory and excitatory synapses may drive blood flow and that suppression of spiking may be as metabolically costly as enhancement of spiking (Logothetis, 2008). (8) Active: Only the most active physiology sites were included in analysis by finding an optimal activity threshold $(0$ to $100 \mathrm{~Hz}$ tested), testing the possibility that metabolic consumption will be more directly related to neurons with higher activity. (9) Selective: Sites were included in analysis by finding an optimal selectivity threshold for positive differences (category 1 preferring) and a second, separate threshold for negative differences (category 2 preferring) ( -200 to $200 \mathrm{~Hz}$ rate difference tested for MUA; -16 to $16 \mu \mathrm{V}$ difference tested for LFP). (10) Gray (white) matter: Sites falling in the gray (white) matter based on anatomical projections were separately tested for their correlation to
fMRI. (11) Posterior/anterior (dorsal/ventral): Sites falling in the lower or upper 50th percentile of AP (DV) coordinates were analyzed separately for their correlation to fMRI. Posterior regions may have more well-defined spatial organization or be less susceptible to fMRI distortion than the anterior pole; alternatively, anterior regions may show stronger category selectivity and more position tolerance leading to less susceptibility to low-level confounds such as eye movements. (12) Concave/ convex: Sites were divided into two groups based on the surface curvature of the gray-white matter boundary nearest to each site. (13) Scale/rotate/translate/all: The 3D neurophysiology coordinates were optimally rescaled in space $(0.8 \times$ to $1.2 \times$ scaling), rotated $(-10$ to 10 degree coronal and sagittal angles), or translated ( -3 to 3 voxels in the $x$, $y$, or $z$ directions). In the "all" condition, all six spatial transform parameters (one scale factor, two rotation angles, and three translations) were simultaneously optimized. (14) Decompress: Given that the electrode compresses the cortical tissue locally, we applied a first order compressive model where the skull provides a rigid boundary condition and compression increases with distance from the skull up to saturation according to a sigmoid function, $\alpha^{\star} r /(\beta+\mathrm{r})$. Both the plateau $\alpha$ and sensitivity $\beta$ of the sigmoid were optimized. (15) Surface: Physiology sites were remapped to the nearest point on the gray-white cortical surface, and correlations were computed with fMRI voxels at those vertex locations. A 2D surface-based analysis has an advantage over computing correlations in native $3 \mathrm{D}$ space as it respects the inherent organization of neural activity along the $2 \mathrm{D}$ cortical manifold and only uses voxels in the vicinity of the gray matter. (16) LFP frequency bands: The power spectrum of 


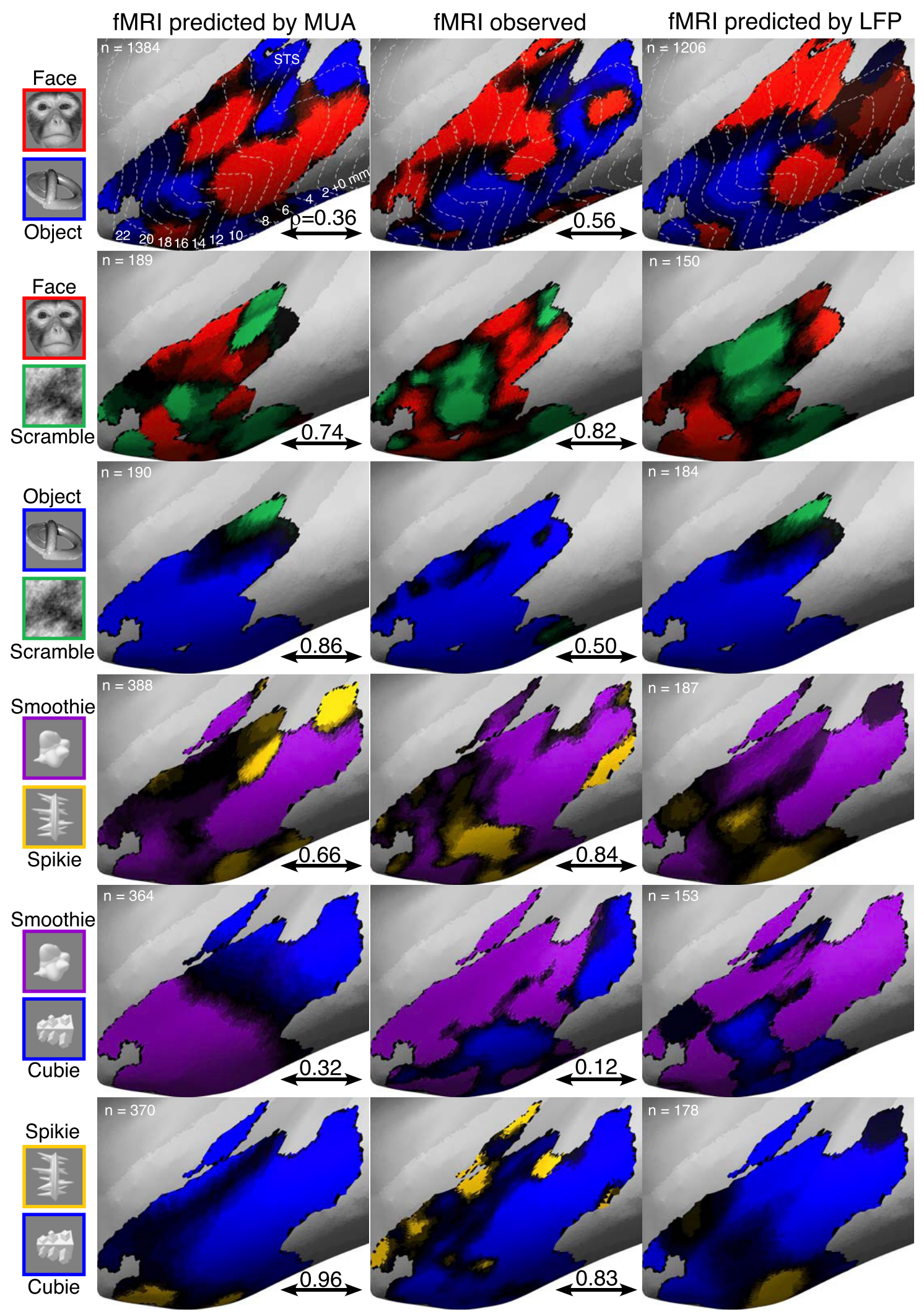

Figure 4. Correspondence between spatially smoothed neurophysiology maps and fMRI in monkey M2. Optimally smoothed MUA (left) and LFP (right) maps are shown for comparison to fMRI contrast maps (middle). The gradients of category selectivity are comparable across maps [e.g., the smoothie vs spikie maps contain a posterior smoothie selective region (purple) followed by a spikie selective zone (yellow)]. However, for one contrast map (smoothies vs cubies), neurophysiology-to-fMRI correlations were low in both subjects (monkey M2 shown here; see Fig. 5 for maps in M1). In these interpolated neurophysiology maps, vertices that were not within $3 \mathrm{~mm}$ of at least three physiology sites were masked, and the same neurophysiology mask was applied to fMRI maps. To aid visual comparison, color values were histogram equalized across all three MUA/LFP/fMRI maps such that colors are not on an absolute scale. 


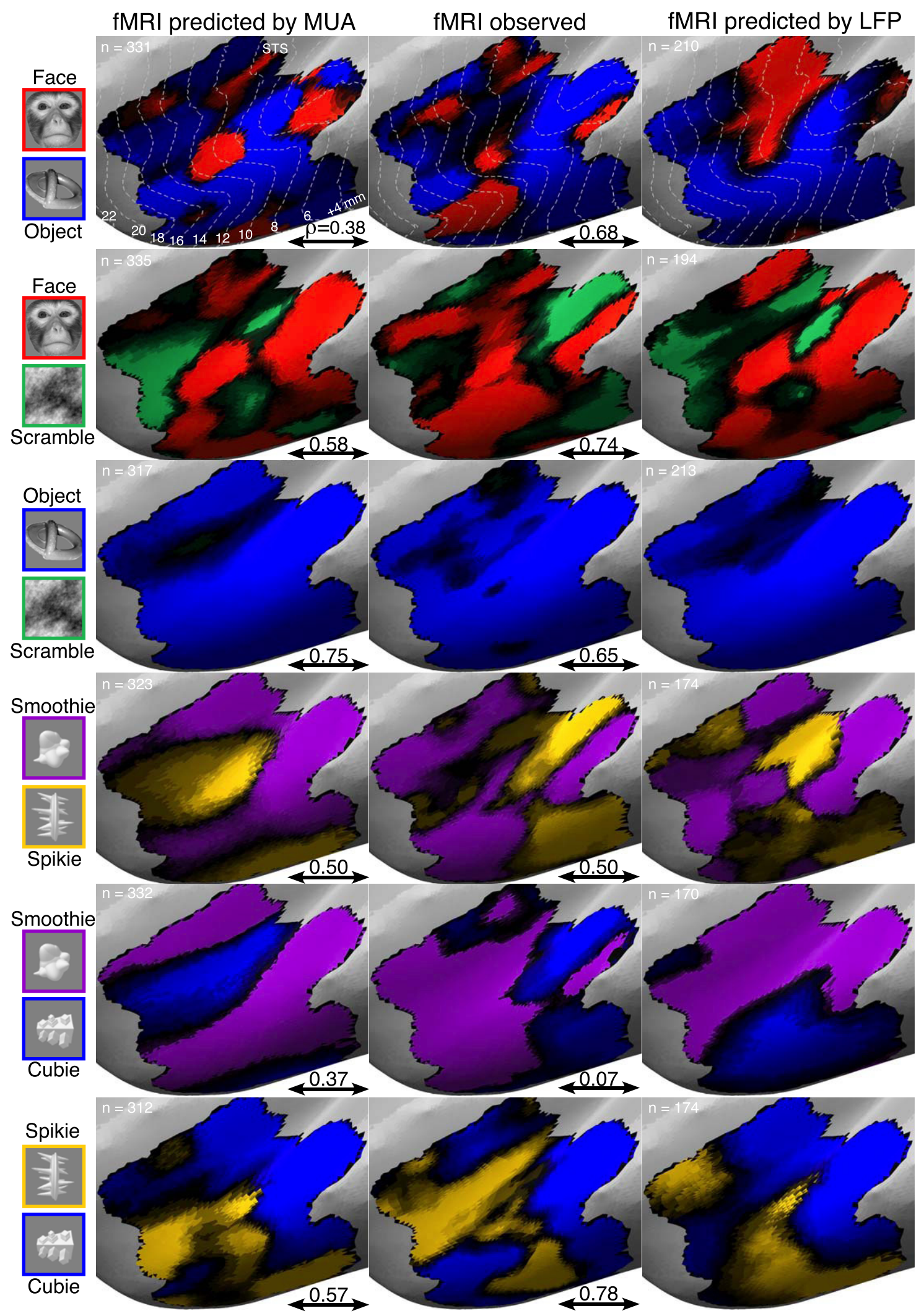

Figure 5. Correspondence between spatially smoothed neurophysiology maps and fMRl in monkey M1. Same format as Figure 4 but for monkey M1.

individual LFP waveforms in a window 50-200 ms following image onset was computed using Welch's moving average method with a window size of 128 samples (sampling rate $=1 \mathrm{kHz}$ ). Average power in six different spectral bands $(0.5-10,16-24,24-40,40-80,80-160$, and $160-300 \mathrm{~Hz})$ was used for comparison to fMRI maps.
In all manipulations requiring optimization of a free parameter [i.e., (1) smoothing, (8) active, (9) selective, (13) scale/rotate/translate/all, and (14) decompression], parameter values were found for half of the sites, and reported correlation values were independently computed on the remaining, withheld half of sites. To ensure a true split of fMRI voxels, 
sites that were sampling the same voxel were forced to be exclusively in the training or testing set and not distributed in both. In general, parameter values were optimized independently for each contrast map in each animal yielding 12 values. We also performed the spatial smoothing analysis using a single smoothing width (median of individual map FWHMs) for all 12 maps and found that this fixed smoothing model gave similar results to individually optimized smoothing kernels (Fig. 6B, rightmost bars).

\section{Statistics}

Spearman's rank correlation was used to compare neurophysiology and fMRI maps. This measure is less susceptible to monotonic nonlinearities (e.g., saturation) in neurophysiologyfMRI relationships; however, similar results were obtained using a linear Pearson correlation coefficient. Since our goal was to predict fMRI category selectivity maps, all correlations were normalized by the maximum achievable explained variance given the reliability of the fMRI maps (Op de Beeck et al., 2008). This normalized correlation measure is termed $\rho$ and is used throughout the text and figures. It will reach a value of 1.0 for a perfect model of fMRI maps. The reliability of fMRI maps was determined by computing, for the voxels that contained physiology sites, the correlation between different runs collected for the same fMRI contrast (split halves approach, $n=30$ iterations) and was corrected for the smaller number of trials using the Spearman Brown correction according to $N^{\star} r /(1+$ $(N-1)^{\star} r$ ) where $N=2$. To estimate a lower bound on the spatial resolution of information in MUA, LFP, and fMRI maps (Fig. 2), high-pass filtered maps were obtained by subtracting smoothed maps from raw maps, and the split halves correlation of the high-passed maps was computed. The point where split halves correlations between high-passed maps dropped below $50 \%$ height provided an estimate of the spatial resolution at which reproducible structure is contained in object selectivity maps.

\section{Results}

\section{Neurophysiological sampling of fMRI volumes}

Our goal was to construct large-scale neurophysiological maps of IT for comparison with fMRI maps obtained previously in the same subjects (Op de Beeck et al., 2008). This entailed sampling across the posterior-to-anterior extent of IT $(\sim 15$ $\mathrm{mm})$ at subvoxel $(<1.25 \mathrm{~mm})$ resolution. MUA and LFP recordings were localized in $3 \mathrm{D}$ with high resolution and in vivo using a stereo microfocal x-ray system developed previously (Cox et al., 2008). Using a common skullbased reference frame, physiology sites were coregistered to functional MRIs for performing direct spatial comparisons (see Materials and Methods). We measured responses to a set of visually-presented images drawn from six categories that included faces and synthetic objects as previous work had shown topographic organization for contrasts of these shape classes
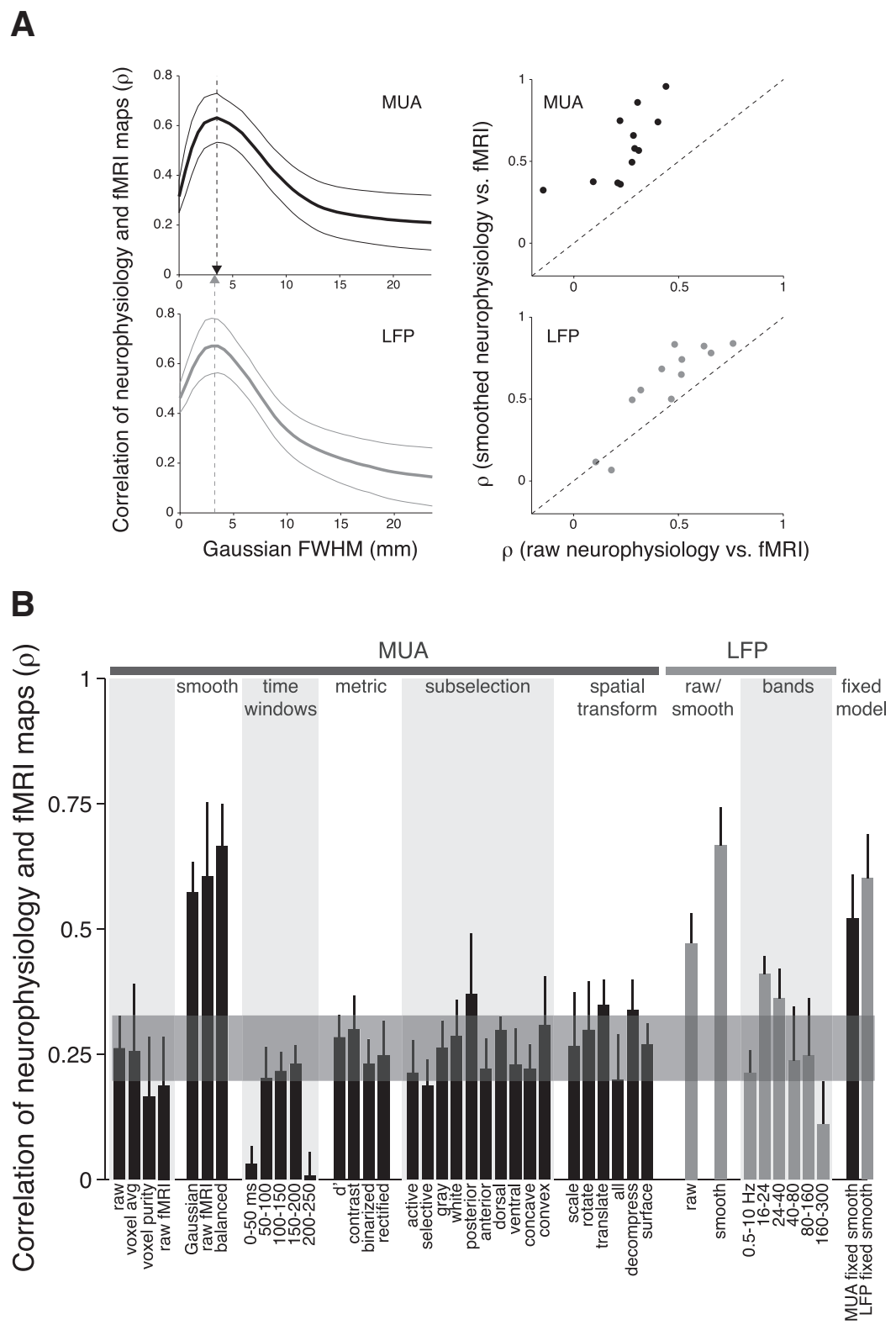

Figure 6. Effect of spatial smoothing and alternative neurophysiology-to-fMRI transforms on neurophysiology-fMRI correlations. $A$, The improvement of neurophysiology-fMRI correlations with spatial smoothing peaked at $3.5 \mathrm{~mm}$ (vertical black dashed line) for MUA maps (upper left) and at $3.2 \mathrm{~mm}$ (vertical gray dashed line) for LFP maps (lower left). Spatial smoothing led to significant improvements in all 12 measured MUA-fMRI correlations (upper right; points scatter above diagonal), but less improvement in LFP-fMRI correlations with smoothing was observed (lower right). B, A number of alternative neurophysiology-tofMRI transforms were tested in addition to spatial smoothing. Transforms could be approximately divided into those that tested different time windows of MUA responses or different frequency bands of the LFP, tested different selectivity metrics, subselected physiology sites, and modified spatial projections of neurophysiology data onto fMRI volumes. In most cases, little improvement was observed for neurophysiology-fMRI correlations above the baseline level computed by correlating raw MUA signals with fMRI (gray band) except when smoothing was applied. The strongest exception is that raw LFP-fMRI correlations began at a higher level than raw MUA-fMRI correlations. However, after spatial smoothing, MUA and LFP correlations with fMRI reached similar levels. Finally, using a fixed smoothing kernel (rightmost bars) gave similar correlations as optimizing smoothing kernels on a per map basis. Error bars reflect SEM across the 12 maps tested.

at the level of fMRI (Tsao et al., 2003; Op de Beeck et al., 2008). Measuring neurophysiological maps using the same object contrasts allowed us to ask whether the structures that appear in fMRI can be explained by neurophysiology maps obtained with the same images. Furthermore, x-ray-based electrode localization allowed us to construct precise neurophysiological maps and extend previous work in IT (Kiani et al., 2007; Bell et al., 2011). 
To illustrate the data used in the analyses below, three representative fMRI voxels are highlighted in Figure $1 A$. In this example, physiology sites sampled within each voxel were tested for their preference for faces versus nonface objects, a contrast that is commonly used in human and monkey fMRI studies (Kanwisher et al., 1997; Tsao et al., 2003). In this particular contrast map, we obtained dense sampling of a few voxels, but across all voxels in the 12 contrast maps collected, two sites were collected per voxel on average (Fig. 1B). An important issue is that neurophysiological maps were constructed serially such that recordings were made on different days and hence under slightly different conditions (e.g., different electrodes) unlike fMRI volumes where all voxels were measured nearly simultaneously. Nonetheless, we found no significant difference in correlations between nearby sites collected on the same day and between nearby sites collected on different days ( $\rho_{\text {within day }}=0.31$ vs $\rho_{\text {between day }}=0.36, p=0.67$, $n_{\text {within day }}=1586, n_{\text {between day }}=1957$, pairs of sites separated by $<500$ micrometers; within penetration distances were calculated using microdrive readings that are of high spatial accuracy $(\sim 1$ $\mu \mathrm{m}$ ), while between-day distances relied on $\mathrm{x}$-ray-based position estimates) with absolute correlation levels similar to those obtained in previous studies comparing patterns of image selectivity across nearby sites (Gochin et al., 1991; Fujita et al., 1992; Kreiman et al., 2006; Kiani et al., 2007). This suggests that we could return to similar neurophysiological locations/conditions across different days with the same accuracy as within the same penetration.

Given that reliable physiological maps could be constructed using our serial approach, an important first question to ask is at what scale do neurophysiology and fMRI maps for each object contrast contain reliable spatial structure. To quantify this, we subtracted smoothed maps from raw maps yielding high-pass filtered maps and asked whether these high-frequency residual maps contained any reproducible structure (i.e., split-half correlation > 0; see Materials and Methods). For MUA and LFP maps, reproducible structure was present down to the smallest scales measurable given the accuracy of the x-ray system $(\sim 200 \mu \mathrm{m})$ while fMRI maps showed little reproducible structure at spatial scales $<2.5 \mathrm{~mm}$ (Fig. 2). Thus, neurophysiological maps of object selectivity contained information at more detailed resolution than fMRI maps, and the goal of this study was to determine the relationship, if any, between information present in neurophysiology and fMRI maps.

The simplest possible model would be that all the physiology sites in a voxel have the same category preference as determined by the fMRI of that voxel. This simple model was clearly false; for example, we found that, even in voxels that were highly face selective, sites within the same voxel had varying preferences for nonface objects versus faces. Across our maps, this heterogeneity led to low overall correlations between site and voxel preferences at the same 3D location (median $\rho=0.26$, average of $n=12$ object contrast maps; $\rho$ refers to the normalized correlation between fMRI and neurophysiology maps when adjusted for noise in fMRI maps, see Materials and Methods). Alternatively, there may exist a mass-action correspondence between the distribution of underlying neurophysiological preferences and the final fMRI "report" of the voxel (i.e., the face selective voxels may contain more face selective sites while the object selective voxels may contain more object selective sites). Perhaps surprisingly, computing this report as the fraction of face/object-preferring sites (termed voxel purity) or the mean selectivity of all sites in each voxel did not lead to any improvement in neurophysiology-fMRI correlations despite averaging out neurophysiological heteroge- neity within a voxel (purity: median $\rho=0.17, p=0.17$; average selectivity: median $\rho=0.26, p=1, n=12$ ). Importantly, voxelbased averaging or purity measures only pool information at a relatively local scale $(1.25 \mathrm{~mm})$ compared with the resolution of reliable information present in fMRI maps (2.5 mm) (Fig. 2), suggesting the need for spatial averaging at larger scales.

\section{Spatial smoothing of neurophysiology maps}

At larger spatial scales, fMRI and neurophysiology maps shared similar structure. For example, the faces versus nonface objects neurophysiology map demonstrated a posterior-to-anterior arrangement of face selective clusters similar to those seen under fMRI (Fig. 3). To test the spatial scale of the relationship between neurophysiology and fMRI, we smoothed neurophysiology maps at different resolutions using a 3D Gaussian kernel. We found that spatially smoothing MUA maps more than doubled MUAfMRI correlations on average $\left(\rho_{\text {smooth }}=0.57\right.$ vs $\rho_{\text {raw }}=0.26, p=$ $0.002, n=12$ ) and produced neurophysiology maps that were often visually difficult to distinguish from fMRI maps across some object contrast maps-even when those object contrast maps were very different from each other (Figs. 4, 5). A fixed smoothing transform (FWHM $=3.5 \mathrm{~mm}$ ) performed as well as a smoothing kernel optimized on a per map basis (Fig. $6 B, \rho_{\text {smooth }}=$ 0.57 vs $\rho_{\text {fixed smooth }}=0.52, p=0.58, n=12$ ). Thus, spatial smoothing was a generic transform that generalized across the particular choice of fMRI contrast whether derived from more natural (e.g., face vs object) or from synthetic (e.g., smoothie vs spikie) object contrasts.

Two possible explanations for this improvement with spatial smoothing are that averaging across sites simply decreased noise inherent to neural firing rates or that smoothing more accurately captured the transform from neurophysiological activity to fMRI signals. Given that neurophysiology maps were already highly reproducible across trial splits such that neural noise was not a major limiting factor $\left(\rho_{\text {MUA,MUA raw }}=0.84\right.$, and pooling more trials had little added benefit, $\rho_{\text {MUA,MUA smooth }}=0.96$ ), we suggest that the primary effect of spatial smoothing was not to reduce noise but to accurately model the fMRI signal. Even when normalizing for the measured improvement in neurophysiology SNR, MUA-fMRI correlations with smoothing remained significantly higher than MUA-fMRI correlations without smoothing (SNR normalized $\rho_{\text {smooth }}=0.49$ vs $\rho_{\text {raw }}=0.25, p=0.003, n=12$ object contrast maps).

MUA maps smoothed at an intermediate scale of 2-5 mm were the best predictors of fMRI maps in all object contrasts that we collected (12 object contrast maps across two monkeys) including those for synthetic objects (i.e., smoothies vs spikies, smoothies vs cubies, and cubies vs spikies) (Fig. 6A). MUA-fMRI correlations peaked at $3.5 \mathrm{~mm}$ smoothing widths (FWHM) (Fig. $6 A$ ), consistent with the scale where fMRI maps begin to contain reliable information (Fig. 2) but not at larger scales that reduced noise but did not accurately model the fMRI signal. In a follow-up analysis, we searched our fMRI maps for local regions $(<2 \mathrm{~mm})$ with high spatial frequency content $(p<0.01$, split halves correlation of highpass-filtered fMRI maps using FWHM = $1.25 \mathrm{~mm}$ ) using a spotlight analysis. Even in these local regions, smoothing MUA maps at 2-5 $\mathrm{mm}$ spatial scales was optimal ( median $=4.7 \mathrm{~mm})$, and no trend between fMRI power at high spatial frequencies and optimal neurophysiology smoothing widths was observed ( $\rho=0.01, p=0.96, n=43$ ), suggesting that stronger higher spatial frequency structure in fMRI did not correspond to the high resolution content in neurophysiology maps. Such an analysis, however, may require improved fMRI signal- 
to-noise ratios at the $1-2 \mathrm{~mm}$ scale as noise may have overwhelmed our fMRI signal at high spatial frequencies even though some information was still present at these frequencies (Fig. 2).

Smoothing fMRI volumes (FWHM $=2.5 \mathrm{~mm}$ ) did significantly average out noise in our fMRI maps increasing their reliability $\left(\rho_{\mathrm{fMRI}, \mathrm{fMRI}}\right.$ unsmoothed $=0.45 \mathrm{vs} \rho_{\mathrm{fMRI}, \mathrm{fMRI}} 2.5 \mathrm{~mm}$ smoothed $=$ $0.78, p=0.001, n=12$ ), and for this reason smoothing is a typical procedure in fMRI studies (Op de Beeck et al., 2008; Tsao et al., 2008b). We used smoothed fMRI maps under the logic that limited information was contained at scales smaller than $2.5 \mathrm{~mm}$ (Fig. 2). However, we also performed analyses using raw fMRI data to determine whether using smoothed fMRI maps may have biased our estimates of the optimal neurophysiology-to-fMRI kernel. We found nearly identical optimal neurophysiology smoothing widths regardless of whether the resulting smoothed physiology map was compared with raw (unsmoothed) fMRI data or smoothed fMRI volumes $\left(\mathrm{FWHM}_{\text {raw }}=4.4\right.$ vs $\mathrm{FWHM}_{2.5 \mathrm{~mm}}=$ $3.5, p=0.91, n=12$ ). And absolute fMRI-neurophysiology correlation levels were similar when using unsmoothed fMRI data as when using smoothed fMRI data after correcting for the reliability of these two different types of fMRI maps ( $\rho_{\text {raw }}=0.61 \mathrm{vs} \rho_{2.5} \mathrm{~mm}=$ $0.57, p=1, n=12$ ).

Finally, we also measured LFP-fMRI correlations across multiple spatial scales. Baseline LFP-fMRI correlations (i.e., without any spatial smoothing) were higher than baseline MUA-fMRI correlations $\left(\rho_{\mathrm{LFP}}=0.47\right.$ vs $\left.\rho_{\mathrm{MUA}}=0.26, p=0.08, n=12\right)$ and only modestly improved with optimal spatial smoothing $\left(\rho_{\text {smooth }}=\right.$ 0.67 vs $\rho_{\text {raw }}=0.47, p=0.11, n=12$ ) (Fig. $6 A$ ). Slightly less spatial smoothing was required for optimal correlation of LFP maps and fMRI maps $(3.2 \mathrm{~mm})$ than was required for optimal correlation of MUA maps and fMRI maps (3.5 mm), although this difference was not significant $(p=0.35, n=12$ ) (Fig. $6 A$ ). Thus, MUA and LFP signals were both highly predictive of fMRI once smoothed at similar $(3.5 \mathrm{~mm})$ scales $\left(\rho_{\mathrm{MUA}}=0.57, \rho_{\mathrm{LFP}}=0.67, p=0.71\right.$, $n=12)$.

\section{Alternative neurophysiology to fMRI transforms}

Besides spatial filtering, many factors may contribute to the mapping from neurophysiology to fMRI signals. For example, although responses may be linear in some regime (Rees et al., 2000), there may be an intervening nonlinearity (e.g., saturation), or fMRI voxels near the gray matter or away from large draining veins in the sulcus may be more reliable indicators of nearby neurophysiological signals (Duong et al., 2001; Goense and Logothetis, 2006; Olman et al., 2007). Also, there may be a slight scaling, rotation, or translation that could improve coregistration between physiology sites and fMRI voxels. Finally, previous work has shown that different aspects of the neural response (e.g., gamma band of the LFP) (Logothetis et al., 2001; Hermes et al., 2012) or different time windows (e.g., early feedforward response vs steady-state firing) (Kim et al., 2004) may better correlate with metabolic signals such as fMRI. To test the potential contribution of these factors, we transformed neurophysiology maps by using different time windows, frequency bands, selectivity metrics, or spatial registrations (see Materials and Methods for complete details). We also varied site and voxel selection criteria to see if neurophysiology-fMRI correlations improved in certain regions. For all transforms tested, we found no significant improvement in neurophysiology-fMRI correlations over baseline levels unlike the significant improvements seen with spatial smoothing of neurophysiology maps (Fig. 6B). Finally, we found no further improvement in neurophysiologyfMRI correlations when cascading the alternative transforms with spatial smoothing (data not shown). Thus, for block design fMRI experiments, the best neurophysiology-to-fMRI model was a $3.5 \mathrm{~mm}$ spatially weighted average of the difference in spike rate evoked by each of the stimulus conditions.

\section{Discussion}

We have reconstructed 3D neurophysiological maps of object selectivity across a wide extent of IT cortex in two monkeys and provided visual and quantitative evidence that spatial gradients of object selectivity in fMRI reflect underlying 3D neural organization at large $(>3.5 \mathrm{~mm})$ spatial scales. Spiking signals, once spatially smoothed, were as predictive of fMRI maps as LFP signals. These results extend previous work by showing that fMRI is a spatially low passed version of neural signals as well as being a temporally low passed version (Logothetis et al., 2001). The widely held belief that fMRI signals are spatially blurred versions of neurophysiology signals (Heeger and Ress, 2002; Op de Beeck, 2010) had only been inferred in previous work (Disbrow et al., 2000; Bell et al., 2011; Hermes et al., 2012) and had yet to be directly tested before this study. Here, we were able to make that direct comparison because we developed and deployed a novel $\mathrm{x}$-ray-based mapping procedure to measure neural maps of object selectivity at finer scales than previous neurophysiological work (Tsao et al., 2006; Bell et al., 2011). With our current x-ray mapping approach, we also found evidence of reproducible neural structure at scales smaller than those resolvable by current fMRI techniques (Fig. 2), a result consistent with previous neurophysiological work (Wang et al., 1996; Tsunoda et al., 2001; Kreiman et al., 2006); whether these currently-fMRI-invisible spatial structures correspond to sharp borders (i.e., modular) or contain additional subregions is an open question for future study.

\section{Limitations of the spatial smoothing model}

Here, we have put forth, for an fMRI block design experiment, our best, current neurophysiology-to-fMRI model: a $3.5 \mathrm{~mm}$ spatially weighted average of the difference in spike rates evoked by each stimulus condition. This model is limited in that as fMRI techniques improve, the exact kernel size is likely to decrease. Furthermore, even for the present data, a spatial smoothing transform did not explain all of the variance in fMRI maps ( $\sim 40 \%$ variance explained) (Fig. $6 B$ ). We used a normalized explained variance measure to account for the reproducibility of data across $\mathrm{fMRI}$ runs that absorbs effects such as subject motion during scanning, differences in MION strength across sessions, or inherent inaccuracy in coregistering functional scans to base anatomical volumes. To further ameliorate these issues, we took precautions such as not considering fMRI volumes when there was excessive movement, searching for optimal affine/deformation transforms, and correcting for magnetic field distortions. Nonetheless, systematic errors may persist depending on the severity of geometric distortions in fMRI maps. One important concern is that fMRI and neurophysiology maps were collected years apart. During this time, the brain may have changed in its anatomical or functional structure. Consistent with a longitudinal study finding little change in macaque brain size after five years of age (Malkova et al., 2006), we found almost no change in brain size in the two monkeys we tested (see Materials and Methods). Furthermore, our previous work showed that functional maps were remarkably stable over the course of a year and over the course of object-specific training (Op de Beeck et al., 2008). In our study, despite the differences between fMRI and neurophysiology methodologies (Table 1; see Materials and Methods), a 


\section{True neurophysiology}
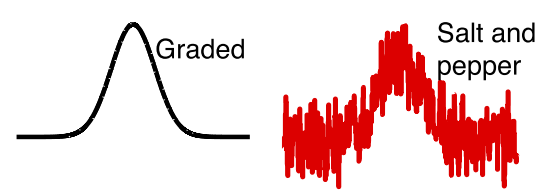

$\square\left[\begin{array}{l}\text { Large } \\ \text { module }\end{array}\right.$

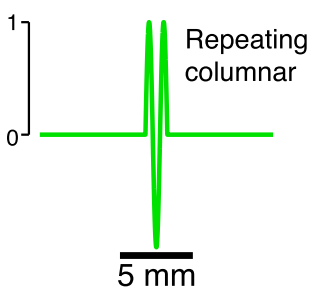

Predicted fMRI

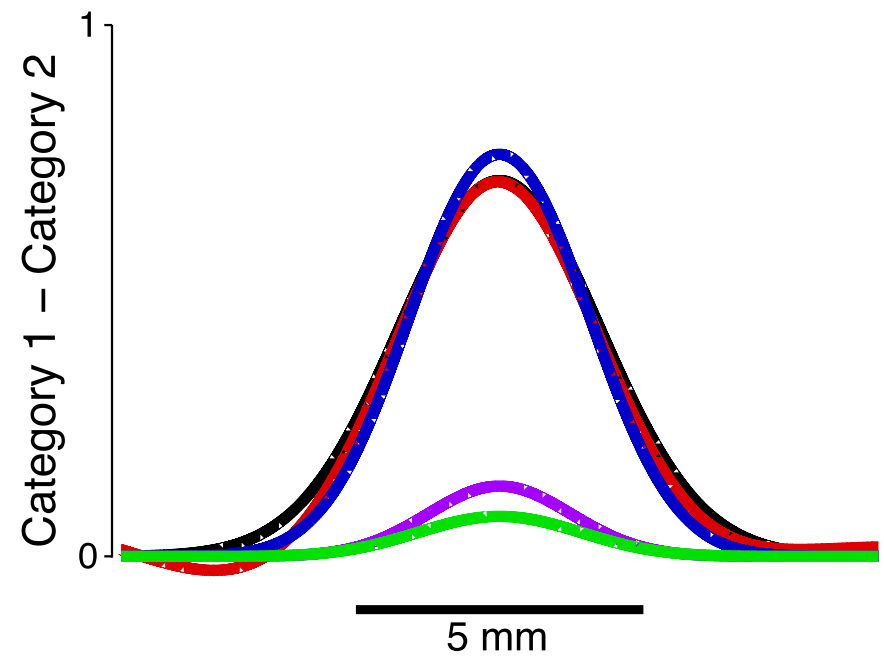

Figure 7. Limitations of fMRI in distinguishing spatial organization at the neurophysiological level. Simulation of how very different putative neurophysiological spatial maps (left) would appear as similar spatial fMRI profiles (right) when smoothed with a $3.5 \mathrm{~mm}$ (FWHM) Gaussian kernel as estimated in this study. Large neurophysiological structures with a graded Gaussian fall-off (black curve, FWHM $=3.5 \mathrm{~mm}$ ) are the most faithfully reflected in both shape and size in fMRI. However, a region with heterogeneous (i.e., "salt-and-pepper") neural selectivity (red, FWHM = $3.5 \mathrm{~mm}$ ) will also appear as a Gaussian in fMRI, and a region with a sharp modular boundary (blue, width $=3.5 \mathrm{~mm}$ ) will have a similar graded profile in fMRI. Thus, graded, sharp, and heterogeneous regions are difficult to distinguish as they have similar amplitude and shape under fMRI. Finally, small single-peaked (purple, width $=0.5 \mathrm{~mm}$ ) regions will appear to be much wider under fMRI although reduced in amplitude, and multi-peaked regions (green, width $=1.5 \mathrm{~mm}, 1$ cycle/mm) will appear as single-peaked in fMRI and also have reduced amplitude.

strong correspondence was observed between these modalities. Nevertheless, our absolute correlation numbers (0.6-0.7 after smoothing) should be taken as a lower bound on the correspondence between neurophysiology and fMRI, and our estimate of the optimal smoothing kernel $(3.5 \mathrm{~mm})$ should be taken as an upper bound.

Another limitation is that viewing each voxel as an identical lowpass filter is possibly an oversimplification. Each voxel contains a unique vascular pattern leading to a complicated spatiotemporal filter that, because of voxel undersampling, potentially aliases high-frequency spatial information into low spatial frequencies (Kriegeskorte et al., 2010). Such a neurovascular filter would allow decoding of information present in high spatial frequencies (Kamitani and Tong, 2005; Haynes and Rees, 2006) with the drawback that neural signals are distributed in space and not easily recoverable using a simple transform. While we do not dispute the possibility of aliasing effects in the fMRI signal, our results show that a large part of the fMRI signal reflects low spatial frequency power in neurophysiology maps—as quantified by our model.

\section{Comparison to previous neurophysiology-fMRI work}

Previous work examining the relationship between neurophysiology and fMRI signals over time instead of across space found that fMRI signals were slightly more correlated to LFP than to MUA signals (Logothetis et al., 2001), but another study found no difference (Kim et al., 2004). In our spatial comparison, the higher correlation of fMRI with LFP signals (Fig. 6A) was nearly eliminated once raw MUA signals were smoothed to broader spatial scales more comparable to LFP and fMRI signals (Fig. 6A). In primary visual cortex, studies have estimated that fMRI maps contain information at the 2-4 mm scale (Shmuel et al., 2007). These estimates were not based on direct neural recordings in V1 but on inference from temporally varying stimuli that leveraged the known architecture of V1 (Engel et al., 1997). Previous estimates of the point spread function in 3T fMRI and 7T fMRI were $3.5 \mathrm{~mm}$ (Parkes et al., 2005) and $2.34 \mathrm{~mm}$ (Shmuel et al., 2007), respectively, providing a lower bound for comparison to our bound of $3.5 \mathrm{~mm}$ obtained with $3 \mathrm{~T}$ fMRI. These numbers do not achieve the biological lower limit set by the hemodynamic response function $(\sim 1 \mathrm{~mm})$ measured in optical imaging studies (Sirotin et al., 2009). So in principle, fMRI can resolve increasingly finer spatial detail if a single plane is imaged instead of the whole brain or if sensitivity is increased for small vessels instead of large veins (Fukuda et al., 2006; Goense and Logothetis, 2006; Goense et al., 2007; Kim and Fukuda, 2008). The primary goal of the present work was not to determine the smallest scale of structure in fMRI maps but to determine the degree to which structure is shared between fMRI maps and underlying neurophysiology. We found that down to the scale where our fMRI maps contained information $(\sim 2.5 \mathrm{~mm})$, these maps were highly predictive of the structures produced by smoothing underlying neurophysiology.

\section{Implications for current fMRI work}

Our technique is comparable to standard fMRI done in humans and monkeys using 3T magnets (Tsao et al., 2003, 2008a; Pitcher et al., 2011). We matched conditions (block design, gradient echo) commonly used while deploying MION, a contrast agent that improves signal-to-noise ratios (Leite et al., 2002) and may improve resolution over more standard BOLD imaging (Kim and Fukuda, 2008; Smirnakis et al., 2007). Our results demonstrate the utility of this standard scanning methodology in revealing the large scale organization in higher level visual areas approaching the current $3.5 \mathrm{~mm}$ limit of fMRI at 3T (Parkes et al., 2005), and our findings may explain why face selective regions in IT (or "face patches") have been consistently detected in fMRI studies (Tsao et al., 2003; Bell et al., 2009; Pinsk et al., 2009; Tsao et al., 2008a). In our previous study, the neurophysiologically determined diameter of the posterior lateral face patch in two monkeys was 3.75 and $5 \mathrm{~mm}$, respectively, and the middle lateral face patch appears to be of similar size or larger (Tsao et al., 2008a; Issa and DiCarlo, 2012). A neurophysiological structure of this size contains energy at spatial frequencies that would easily pass through the spatial smoothing kernel determined in this study (Gaussian, FWHM = 
$3.5 \mathrm{~mm}$ ) and would be visible in fMRI at a size similar to its true underlying size (Fig. 7).

However, a more precise description of the neural organization of face versus object maps given our observations (Fig. 3 ) is that they contain salt-and-pepper structure locally as well as biases in face selectivity at large scales. Under this biased salt-andpepper model, fMRI smoothes out local heterogeneity but provides an accurate picture of large-scale face selectivity gradients. We speculate that the contrast (Smoothies vs Cubies; Figs. 4, 5) that gave the weakest correlations in both monkeys may have contained weak large-scale structure possibly because smoothies and cubies may be similar in shape space.

Finally, we have only shown that neurophysiology maps contain reliable structure at current fMRI-invisible scales in the statistical sense and have not provided an explicit description (e.g., columnar) of these fine scale structures. To do so would require denser, more even sampling and may require using different images that expose fine scale organization. Our spatial blurring model predicts that many other patterns of neurophysiological organization besides salt-and-pepper organization would appear to have a similar spatial fMRI profile as a large face selective region. For example, our models predict that signals that change selectivity rapidly across space (i.e., repeated motifs at fine scales such as orientation columns) will appear as relatively pure in fMRI as will impure (salt-and-pepper) regions (Fig. 7). Also, it predicts that a region of highly pure selectivity (module) much smaller than a face patch $(0.5 \mathrm{~mm})$ will appear to be much larger in $\mathrm{fMRI}(>3 \mathrm{~mm})$ and could be confused with a large face patch (Fig. 7). These effects of spatial smoothing suggest cautious interpretation of regions of interest or "modules" when localized using $\mathrm{fMRI}$, as many of the possible underlying neurophysiological patterns are not "modular" in the sense that they can be far from pure and need not have well-defined boundaries. Nonetheless, fMRI maps of object selectivity should be considered rough but still invaluable guides to the spatial organization of neural selectivity in IT.

\section{References}

Attwell D, Buchan AM, Charpak S, Lauritzen M, Macvicar BA, Newman EA (2010) Glial and neuronal control of brain blood flow. Nature 468:232243. CrossRef Medline

Bell AH, Hadj-Bouziane F, Frihauf JB, Tootell RB, Ungerleider LG (2009) Object representations in the temporal cortex of monkeys and humans as revealed by functional magnetic resonance imaging. J Neurophysiol 101: 688-700. CrossRef Medline

Bell AH, Malecek NJ, Morin EL, Hadj-Bouziane F, Tootell RB, Ungerleider LG (2011) Relationship between functional magnetic resonance imaging-identified regions and neuronal category selectivity. J Neurosci 31:12229-12240. CrossRef Medline

Cox DD, Papanastassiou AM, Oreper D, Andken BB, Dicarlo JJ (2008) High-resolution three-dimensional microelectrode brain mapping using stereo microfocal x-ray imaging. J Neurophysiol 100:2966-2976. CrossRef Medline

De Baene W, Premereur E, Vogels R (2007) Properties of Shape Tuning of Macaque Inferior Temporal Neurons Examined Using Rapid Serial Visual Presentation. J Neurophysiol 97:2900-2916. CrossRef Medline

Disbrow EA, Slutsky DA, Roberts TP, Krubitzer LA (2000) Functional MRI at 1.5 tesla: a comparison of the blood oxygenation level-dependent signal and electrophysiology. Proc Natl Acad Sci U S A 97:9718-9723. CrossRef Medline

Duong TQ, Kim DS, Uğurbil K, Kim SG (2001) Localized cerebral blood flow response at submillimeter columnar resolution. Proc Natl Acad Sci U S A 98:10904-10909. CrossRef Medline

Edwards R, Xiao D, Keysers C, Földiák P, Perrett D (2003) Color sensitivity of cells responsive to complex stimuli in the temporal cortex. J Neurophysiol 90:1245-1256. CrossRef Medline

Engel SA, Glover GH, Wandell BA (1997) Retinotopic organization in hu- man visual cortex and the spatial precision of functional MRI. Cereb Cortex 7:181-192. CrossRef Medline

Fujita I, Tanaka K, Ito M, Cheng K (1992) Columns for visual features of objects in monkey inferotemporal cortex. Nature 360:343-346. CrossRef Medline

Fukuda M, Moon CH, Wang P, Kim SG (2006) Mapping iso-orientation columns by contrast agent-enhanced functional magnetic resonance imaging: reproducibility, specificity, and evaluation by optical imaging of intrinsic signal. J Neurosci 26:11821-11832. CrossRef Medline

Gochin PM, Miller EK, Gross CG, Gerstein GL (1991) Functional interactions among neurons in inferior temporal cortex of the awake macaque. Exp Brain Res 84:505-516. Medline

Goense JB, Logothetis NK (2006) Laminar specificity in monkey V1 using high-resolution SE-fMRI. Magn Reson Imaging 24:381-392. CrossRef Medline

Goense JBM, Zappe AC, Logothetis NK (2007) High-resolution fMRI of macaque V1. Magn Reson Imaging 25:740-747. CrossRef Medline

Haynes JD, Rees G (2006) Decoding mental states from brain activity in humans. Nat Rev Neurosci 7:523-534. Medline

He BJ, Snyder AZ, Zempel JM, Smyth MD, Raichle ME (2008) Electrophysiological correlates of the brain's intrinsic large-scale functional architecture. Proc Natl Acad Sci U S A 105:16039-16044. CrossRef Medline

Heeger DJ, Ress D (2002) What does fMRI tell us about neuronal activity? Nat Rev Neurosci 3:142-151. CrossRef Medline

Hermes D, Miller KJ, Vansteensel MJ, Aarnoutse EJ, Leijten FS, Ramsey NF (2012) Neurophysiologic correlates of fMRI in human motor cortex. Hum Brain Mapp 33:1689-1699. CrossRef Medline

Hubel DH, Wiesel TN (1974) Sequence regularity and geometry of orientation columns in the monkey striate cortex. J Comp Neurol 158:267-293. CrossRef Medline

Hung CP, Kreiman G, Poggio T, DiCarlo JJ (2005) Fast readout of object identity from macaque inferior temporal cortex. Science 310:863-866. CrossRef Medline

Issa EB, DiCarlo JJ (2012) Precedence of the eye region in neural processing of faces. J Neurosci 32:16666-16682. CrossRef Medline

Issa EB, Papanastassiou AM, Andken BB, DiCarlo JJ (2010) Towards largescale, high resolution maps of object selectivity in inferior temporal cortex. Front Neurosci Conference Abstract: Computational and Systems Neuroscience, Salt Lake City, UT. doi: 10.3389/conf.fnins.2010.03.00154. CrossRef

Kamitani Y, Tong F (2005) Decoding the visual and subjective contents of the human brain. Nat Neurosci 8:679-685. CrossRef Medline

Kanwisher N, McDermott J, Chun MM (1997) The fusiform face area: a module in human extrastriate cortex specialized for face perception. J Neurosci 17:4302-4311. Medline

Kiani R, Esteky H, Mirpour K, Tanaka K (2007) Object category structure in response patterns of neuronal population in monkey inferior temporal cortex. J Neurophysiol 97:4296-4309. CrossRef Medline

Kim DS, Duong TQ, Kim SG (2000) High-resolution mapping of isoorientation columns by fMRI. Nat Neurosci 3:164-169. CrossRef Medline

Kim DS, Ronen I, Olman C, Kim SG, Ugurbil K, Toth LJ (2004) Spatial relationship between neuronal activity and BOLD functional MRI. Neuroimage 21:876-885. CrossRef Medline

Kim SG, Fukuda M (2008) Lessons from fMRI about mapping cortical columns. Neuroscientist 14:287-299. CrossRef Medline

Kreiman G, Hung CP, Kraskov A, Quiroga RQ, Poggio T, DiCarlo JJ (2006) Object selectivity of local field potentials and spikes in the macaque inferior temporal cortex. Neuron 49:433-445. CrossRef Medline

Kriegeskorte N, Cusack R, Bandettini P (2010) How does an fMRI voxel sample the neuronal activity pattern: compact-kernel or complex spatiotemporal filter? Neuroimage 49:1965-1976. CrossRef Medline

Ku SP, Tolias AS, Logothetis NK, Goense J (2011) fMRI of the faceprocessing network in the ventral temporal lobe of awake and anesthetized macaques. Neuron 70:352-362. CrossRef Medline

Leite FP, Tsao D, Vanduffel W, Fize D, Sasaki Y, Wald LL, Dale AM, Kwong KK, Orban GA, Rosen BR, Tootell RB, Mandeville JB (2002) Repeated fMRI Using Iron Oxide Contrast Agent in Awake, Behaving Macaques at 3 Tesla. Neuroimage 16:283-294. CrossRef Medline

Logothetis NK (2008) What we can do and what we cannot do with fMRI. Nature 453:869-878. CrossRef Medline

Logothetis NK, Pauls J, Augath M, Trinath T, Oeltermann A (2001) Neuro- 
physiological investigation of the basis of the fMRI signal. Nature 412: 150-157. CrossRef Medline

Malkova L, Heuer E, Saunders RC (2006) Longitudinal magnetic resonance imaging study of rhesus monkey brain development. Eur J Neurosci 24: 3204-3212. CrossRef Medline

Masamoto K, Kanno I (2012) Anesthesia and the quantitative evaluation of neurovascular coupling. J Cereb Blood Flow Metab 32:1233-1247. CrossRef Medline

Mur M, Ruff DA, Bodurka J, De Weerd P, Bandettini PA, Kriegeskorte N (2012) Categorical, yet graded-single-image activation profiles of human category-selective cortical regions. J Neurosci 32:8649-8662. CrossRef Medline

Ohki K, Chung S, Ch'ng YH, Kara P, Reid RC (2005) Functional imaging with cellular resolution reveals precise micro-architecture in visual cortex. Nature 433:597-603. CrossRef Medline

Olman CA, Inati S, Heeger DJ (2007) The effect of large veins on spatial localization with GE BOLD at 3 T: Displacement, not blurring. Neuroimage 34:1126-1135. CrossRef Medline

Op de Beeck HP (2010) Against hyperacuity in brain reading: Spatial smoothing does not hurt multivariate fMRI analyses? Neuroimage 49: 1943-1948. CrossRef Medline

Op de Beeck HP, Deutsch JA, Vanduffel W, Kanwisher NG, DiCarlo JJ (2008) A stable topography of selectivity for unfamiliar shape classes in monkey inferior temporal cortex. Cereb Cortex 18:1676-1694. Medline

Parkes LM, Schwarzbach JV, Bouts AA, Deckers RH, Pullens P, Kerskens CM, Norris DG (2005) Quantifying the spatial resolution of the gradient echo and spin echo BOLD response at 3 tesla. Magn Reson Med 54:14651472. CrossRef Medline

Pinsk MA, Arcaro M, Weiner KS, Kalkus JF, Inati SJ, Gross CG, Kastner S (2009) Neural representations of faces and body parts in macaque and human cortex: a comparative fMRI study. J Neurophysiol 101:2581-2600. CrossRef Medline

Pitcher D, Dilks DD, Saxe RR, Triantafyllou C, Kanwisher N (2011) Differential selectivity for dynamic versus static information in face-selective cortical regions. Neuroimage 56:2356-2363. CrossRef Medline

Rees G, Friston K, Koch C (2000) A direct quantitative relationship between the functional properties of human and macaque V5. Nat Neurosci 3:716-723. CrossRef Medline
Shmuel A, Yacoub E, Chaimow D, Logothetis NK, Ugurbil K (2007) Spatiotemporal point-spread function of fMRI signal in human gray matter at 7 Tesla. Neuroimage 35:539-552. CrossRef Medline

Sirotin YB, Hillman EM, Bordier C, Das A (2009) Spatiotemporal precision and hemodynamic mechanism of optical point spreads in alert primates. Proc Natl Acad Sci U S A 106:18390-18395. CrossRef Medline

Smirnakis SM, Schmid MC, Weber B, Tolias AS, Augath M, Logothetis NK (2007) Spatial specificity of BOLD versus cerebral blood volume fMRI for mapping cortical organization. J Cereb Blood Flow Metab 27: 1248-1261. CrossRef Medline

Tanigawa H, Lu HD, Roe AW (2010) Functional organization for color and orientation in macaque V4. Nat Neurosci 13:1542-1548. Medline

Tsao DY, Freiwald WA, Knutsen TA, Mandeville JB, Tootell RB (2003) Faces and objects in macaque cerebral cortex. Nat Neurosci 6:989-995. CrossRef Medline

Tsao DY, Freiwald WA, Tootell RB, Livingstone MS (2006) A cortical region consisting entirely of face-selective cells. Science 311:670-674. CrossRef Medline

Tsao DY, Moeller S, Freiwald WA (2008a) Comparing face patch systems in macaques and humans. Proc Natl Acad Sci U S A 105:19514-19519. CrossRef Medline

Tsao DY, Schweers N, Moeller S, Freiwald WA (2008b) Patches of faceselective cortex in the macaque frontal lobe. Nat Neurosci 11:877-879. CrossRef Medline

Tsunoda K, Yamane Y, Nishizaki M, Tanifuji M (2001) Complex objects are represented in macaque inferotemporal cortex by the combination of feature columns. Nat Neurosci 4:832-838. CrossRef Medline

Vul E, Lashkari D, Hsieh PJ, Golland P, Kanwisher N (2012) Data-driven functional clustering reveals dominance of face, place, and body selectivity in the ventral visual pathway. J Neurophysiol 108:2306-2322. CrossRef Medline

Wang G, Tanaka K, Tanifuji M (1996) Optical imaging of functional organization in the monkey inferotemporal cortex. Science 272:1665-1668. CrossRef Medline

Yacoub E, Harel N, Ugurbil K (2008) High-field fMRI unveils orientation columns in humans. Proc Natl Acad Sci U S A 105:10607-10612. CrossRef Medline 